\title{
Videojuegos, historia y patrimonio: primeros resultados de una investigación educativa evaluativa en educación secundaria
}

\section{Video games, history and heritage: first results of an educational evaluation research in secondary education}

\author{
Alejandro Egea Vivancos \\ Universidad de Murcia \\ alexegea@um.es \\ Laura Arias Ferrer \\ Universidad de Murcia \\ larias@um.es \\ Alfonso J. García López \\ Fundación Integra \\ alfonso.garcia@f-integra.org
}

Recibido: 20/02/2017

Aceptado: 08/05/2017

Publicado: 30/06/2017

\section{RESUMEN}

Entre las misiones de la Fundación Integra está el acelerar el proceso de implantación de la Sociedad de la Información en la Región de Murcia, por lo que focaliza una gran parte de su actividad en la generación de múltiples recursos digitales. En el marco de esta línea de actuación se desarrolló en el año 2015 el videojuego de realidad virtual "El Misterio de la Encomienda de Ricote". Se trata de un contenido interactivo al cual se juega mediante gafas Oculus Rift. Este videojuego está dirigido principalmente a escolares de Educación Secundaria, en concreto a estudiantes de 2. ESO a 4.. ESO, tanto por el contenido a tratar como por las dinámicas de juego diseñadas. A pesar de que son ya bastante numerosas las propuestas educativas para introducir la historia a través de los videojuegos, en raras ocasiones se procede a una evaluación educativa del videojuego. Aunque en este caso la jugabilidad del producto se demostró bastante alta en diferentes ocasiones, se hacía necesaria una reflexión sobre las ventajas didácticas que este juego podía proporcionar. La evaluación consta de una primera fase de prepilotaje para la que se ha diseñado un primer cuestionario con siete ítems que permiten un análisis de tipo mixto, que ha sido aplicado a modo de prueba piloto en un centro de Educación Secundaria sobre una muestra de 14 individuos de 13-14 años. Gracias a este instrumento, se procura precisar cuáles de las pruebas que se desarrollan durante el juego son más eficaces para el aprendizaje. Así mismo, la evaluación se completa con un segundo instrumento que analiza el nivel de aceptación por parte de los jugadores así como su percepción sobre lo aprendido.

\section{PALABRAS CLAVE}

Enseñanza de la historia; Educación Patrimonial; Juegos Educativos; Videojuegos; TIC y Educación. 


\section{ABSTRACT}

Among the main objectives of Fundación Integra are those related to the implementation of New Technologies and the development of the Information Society in the Region of Murcia. To get these aims, this foundation focuses its attention and its efforts on the production of a wide range of digital resources. As part of this course of action, it was developed in 2015 a virtual reality gaming experience called "El Misterio de la Encomienda de Ricote". This interactive content is presented with immersive technology by using Oculus Rift glasses. This video game is directed towards Secondary Education students, and specifically towards $2^{\text {nd }}$ and $4^{\text {th }}$ grades, not only because of the main content addressed in the game (end of the $15^{\text {th }}$ century-beginning of the $16^{\text {th }}$ century) but also because of the game strategies designed. It is not unusual to find several proposals based on the introduction of video games in the teaching practice. However, it is scarcely developed an educational assessment of such a resource and its benefits for the teaching and learning process. In this sense, although the playability of the product was considered to be quite appropriate, and the level of satisfaction among the participants was high, it was necessary to assess the educational benefits that the game could provide by itself. To answer these questions, it was designed a first questionnaire (as pre- and post-testing) with seven items which was piloted with a sample comprised by 14 students in the age range of 13 and 14. Thanks to this instrument, it was possible to determine the educational effectiveness of the strategies developed during the process. This first results were compared with the own perception of the students about the gaming experience, thanks to a second qualitative questionnaire where the students could express their impressions about the game and about the own educational process.

\section{KEYWORDS}

History Instruction; Heritage Education; Educational Games; Video Games; Technology Uses in Education.

\section{CITA RECOMENDADA}

Egea, A., Arias, L. y García, A.J. (2017). Videojuegos, historia y patrimonio: primeros resultados de una investigación educativa evaluativa en educación secundaria. RIITE. Revista Interuniversitaria de Investigación en Tecnología Educativa, 2, 28-40. Doi: http://dx.doi.org/10.6018/riite/2017/283801

\section{Principales aportaciones del artículo y futuras líneas de investigación:}

- Se realiza la evaluación de un videojuego desde el punto de vista de qué aprenden los estudiantes.

- Se identifican qué tipo de pruebas favorecen mejor el recuerdo de los contenidos.

- En el futuro se podrán extraer los datos de la fase de pilotaje, ampliando la muestra. 


\section{INTRODUCCIÓN}

Desde su creación en 1998, la Fundación Integra ha tenido como una de sus tareas principales favorecer la implantación de la Sociedad de la Información en la Región de Murcia. Siguiendo esta línea, dicha institución concentra buena parte de sus acciones en torno a la creación de numerosos recursos digitales, centrados especialmente en favorecer la difusión del patrimonio regional entre los más jóvenes. Para ello se vienen desarrollando desde entonces diferentes producciones de las que, quizás, se podría destacar el largometraje de animación "Carthago Nova" que llegó a estar nominado como mejor película de animación en los Premios Goya de 2012 (Pérez y Molina, 2011). Sin embargo, esta producción no es más que la punta de lanza de un copioso número de materiales, todos ellos disponibles en el portal Región de Murcia Digital (regmurcia.com).

Siguiendo esta línea de trabajo en el 2015 se produjo un videojuego de realidad virtual inmersiva titulado "El Misterio de la Encomienda de Ricote" (Escribano-Miralles, 2016). La producción corrió a cargo de la Fundación Integra que encargó su desarrollo a las empresas Estudio Future (programación) y Patrimonio Inteligente (gamificación y asesoramiento histórico). A través de unos contenidos interactivos y gamificados, a los que se puede jugar con gafas Oculus Rift, se presenta la historia del edificio del mismo nombre de la localidad de Ricote. El juego, y el vídeo introductorio que lo acompaña, da a conocer la historia del edificio construido a finales del siglo XV en la citada localidad. Aunque cualquier persona puede jugar, su público potencial son los escolares de Educación Secundaria, en concreto estudiantes de 2.ำ ESO a $4 .$. ESO, ya que los contenidos son más fácilmente adaptables a los propios de las Ciencias Sociales en estos cursos concreto (Decreto 220/2015). Las dinámicas de juego diseñadas también tuvieron en cuenta estas edades.

Además de las propias pruebas que se realizaron durante la producción, el juego "El Misterio de la Encomienda de Ricote" fue probado en una decena de centros de Educación Secundaria de toda la Región de Murcia, y ha sido ofrecido a todo aquel interesado en la Semana de la Ciencia de Murcia o en una campaña estival por las playas regionales. Todo ello, permitió constatar que la usabilidad y jugabilidad del producto era bastante alta. Sin embargo, de cara a posibles diseños de juegos similares, se planteó la necesidad de reflexionar sobre las aportaciones y ventajas que este juego proporcionaba en concreto a la hora de enseñar historia y llevar el patrimonio a las aulas. Al fin y al cabo el objetivo final de esta y similares iniciativas es que pueda servir como recurso en el proceso de enseñanza-aprendizaje.

Por todo ello, fruto de la colaboración entre la Fundación Integra y la Universidad de Murcia, se inició un proceso de evaluación del producto que, en suma, pretendía responder a la siguiente cuestión: ¿En qué medida se aprendía la historia de la Encomienda de Ricote con este juego? En efecto, la intención era medir la efectividad que presentaba este recurso concreto para la transmisión de conocimientos al alumnado. De este modo, sería posible aplicar las conclusiones obtenidas en futuros desarrollos similares de la fundación, cumpliendo con los intereses de los docentes y los gustos o necesidades educativas de los estudiantes.

\section{ENSEÑANZA DE LA HISTORIA Y VIDEOJUEGOS}

La relación entre la enseñanza y la utilización de los videojuegos es una cuestión que ya posee cierta tradición bibliográfica. Desde su aparición a finales de los años 40 del siglo XX, su empleo para diversos fines, incluido el educativo, ya cuenta con bastante reconocimiento (Barbier, 2014). Es evidente que el simple hecho de usar videojuegos conlleva el aprendizaje per se, ya que siempre se aprende algo con el juego o de manera colateral a él (Becker, 2011) 
y que jugar también favorece y trabaja un nuevo tipo de alfabetización imprescindible en el mundo actual (Gee, 2007). ¿Pero se puede aprender historia con ellos? La oferta de videojuegos de corte histórico que ofrece el mercado es constante y variada, ya sean sobre el medievo (Jiménez, 2016) u otras épocas (Rivero, 2009), que además introducen variados elementos del patrimonio cultural en sus escenarios que posibilita su incorporación al aula (Anderson et al., 2010). Por esa razón, muchos docentes han procurado introducir ciertos períodos históricos en sus aulas a través de este recurso. En efecto, ya son varias las propuestas creadas desde el aula o para el aula que reflexionan sobre las ventajas y animan a su uso (Grup F9, 1998; Jiménez-Palacios y Cuenca, 2016; Téllez e Iturriaga, 2014). Ya sea porque proporcionan un aprendizaje significativo o duradero (Ayén, 2010), porque permiten resolver problemas sociales o históricos relevantes (Cuenca y Martín, 2010), porque sirven para desarrollar y experimentar representaciones y demostraciones históricas (Moreno, 2010), por su capacidad de facilitar la comprensión de conceptos complejos que requieren de cierta abstracción (Cuenca, 2001) como son los contextos espacio-temporales (Gálvez, 2006) o, simplemente, por su capacidad de aumentar la motivación (Etxeberria, 2001).

Sin embargo, el lector interesado se encontrará con cierta dificultad a la hora de encontrar investigaciones empíricas que demuestren con datos todas estas ventajas o, más aún, si la aplicación de este recurso llegó a tener algún tipo de repercusión en el aprendizaje de los estudiantes (Del Moral et al. 2012; Evaristo et al. 2016). En efecto, a pesar de que son ya muchas las propuestas educativas que acercan la historia a través de los videojuegos, pocas veces se ha desarrollado una evaluación del videojuego desde un punto de vista educativo. De hecho, es difícil encontrar propuestas metodológicas de evaluación de videojuegos, salvo ciertas excepciones como el denominado 4PEG, The Four Pillars of Educational Games (Beckert, 2011). Por todo ello, se hace más valiosa la presentación de los resultados de los primeros pasos de la investigación que aquí se presenta.

\section{MÉTODO}

\subsection{Objetivos}

Esta investigación responde a una serie de objetivos, encaminados todos ellos a analizar las ventajas educativas que conlleva el uso de videojuegos en las aulas, concretamente de historia. Por un lado, se pretende (1) identificar qué contenidos de los desarrollados en el juego "El Misterio de la Encomienda de Ricote" son recordados por los estudiantes/jugadores para, en segundo lugar, (2) analizar qué tipo de pruebas son más propicias a que se recuerden los contenidos y, por último, (3) conocer las valoraciones de los estudiantes tras el juego desde un punto de vista lúdico y educativo.

\subsection{Diseño de la investigación}

Para dar respuesta a estos objetivos se procedió a diseñar una investigación evaluativa de corte mixto (McMillan y Schumacher, 2005). Gracias a los instrumentos de evaluación diseñados se pretendía poder explicar y controlar el fenómeno objeto de estudio. Sin embargo, antes de proceder a la investigación con una muestra mayor era necesario establecer un estudio pre-piloto que sirviera en sí mismo de validación del instrumento.

\subsection{Contexto y participantes}

El contexto elegido son los estudiantes de 3. de Educación Secundaria Obligatoria ya que es en este curso en el que se trabaja el periodo histórico en el que se enmarca el juego, los 
inicios de la Edad Moderna. Para la fase del estudio pre-piloto han participado 14 estudiantes $(\mathrm{m}=7, \mathrm{f}=7)$ de un centro público de Secundaria de la localidad de Molina de Segura, tratándose por tanto de un muestreo no probabilístico por conveniencia (McMillan y Schumacher, 2005). En cuanto al marco de la actividad, cabe decir que en un aula-clase todo el grupo cumplimentó el instrumento $\mathrm{C} 1$ por primera vez y fue proyectado el vídeo. Se les animó a los estudiantes a colaborar pues de sus opiniones y sinceridad saldría beneficiado el propio juego. El hecho de comentarles que eran los "probadores" del juego resultó eficaz pues su colaboración fue excelente. Posteriormente, de manera individual, cada estudiante accedía a otra sala en la que se encontraba el puesto de juego. Tras jugar, cumplimentaban por segunda vez el instrumento C1 y cerraban la evaluación con el instrumento $\mathrm{C} 2$.

\subsection{Recogida de información: instrumentos y procedimiento}

La evaluación general estaba integrada por una primera fase de prepilotaje para la que se diseñaron dos instrumentos ex novo. Por un lado, para responder a los objetivos 1 y 2 (¿qué se recuerda tras jugar?; ¿qué contenidos son mejor recordados?) se cuenta con un primer cuestionario que incluyó siete ítems de respuesta abierta que permite un análisis de tipo cuantitativo, pues las respuestas se procesan en base a su corrección (C1). Este cuestionario se aplicó en dos fases diferenciadas: antes del inicio de la acción educativa diseñada (visionado del vídeo introductorio y juego), a modo de pre-test o prueba de entrada, y una vez finalizada la acción educativa completa, contando así con un post-test o evaluación de salida. Con el empleo de este instrumento, se consiguió determinar qué contenidos recordaban los estudiantes tras finalizar la acción educativa, y cuáles de las fases o pruebas que se desarrollan durante la acción (vídeo y juego) son más eficaces a la hora de recordar los contenidos de tipo histórico. Por ello, el cuestionario recoge contenidos que aparecen en el vídeo de introducción al juego, contenidos que aparecen exclusivamente en el juego, contenidos que aparecen en ambos elementos y, como herramienta de control, contenidos que no aparecen en ningún momento (Tabla 1).

Tabla 1

Ítems del cuestionario C1. Se excluyen aquellos referidos a la identificación

Ítem Enunciado

$1 \quad$ ¿Podrías decirnos cuál de estos mapas representa mejor la situación de Ricote?

2 En el vídeo y juego se habla de la Casa de la Encomienda de Ricote. ¿Sabes lo que era? ¿Para qué pudo servir?

3 ¿Cuándo se construyó la Casa de la Encomienda de Ricote?

$4 \quad$ ¿A qué Orden pertenecía?

$5 \quad$ ¿Cuál era el escudo de esa Orden? Rodea con un círculo la letra que se corresponda con el escudo que creas correcto.

$6 \quad$ ¿Quiénes eran los moriscos?

$7 \quad$ ¿Sabrías decirnos quiénes fueron estos personajes? 
Así mismo, para responder al objetivo 3 de la evaluación (¿cuál es la valoración de los estudiantes respecto al juego?) se diseñó un segundo instrumento (C2) que procuraba determinar el nivel de aceptación por parte de los estudiantes (jugadores), tanto del vídeo como del juego, así como su percepción sobre lo que habían aprendido con esta acción. En este caso se han combinado preguntas de escala Likert con preguntas de respuesta abierta, por lo que la investigación es de tipo mixta. Este cuestionario fue cumplimentado por el alumnado una vez finalizada la acción educativa diseñada (Tabla 2).

Tabla 2

Ítems del cuestionario C2. Se excluyen aquellos referidos a la identificación

\begin{tabular}{|c|c|c|}
\hline Ítem & Enunciado & Tipo de pregunta \\
\hline 1 & ¿Qué he aprendido hoy......durante el vídeo? & Respuesta abierta. \\
\hline 2 & ¿Qué he aprendido hoy......durante el juego? & Respuesta abierta. \\
\hline 3 & $\begin{array}{c}\text { ¿Crees que conoces mejor la historia de la Encomienda de } \\
\text { Ricote? Rodea con un círculo tu respuesta. }\end{array}$ & $\begin{array}{c}\text { Nada / Muy poco / Poco } \\
\text { / Bastante / Mucho / } \\
\text { Muchísimo }\end{array}$ \\
\hline 4 & $\begin{array}{c}\text { ¿Te ha gustado la experiencia? Rodea con un círculo tu } \\
\text { respuesta. }\end{array}$ & $\begin{array}{c}\text { Nada / Muy poco / Poco } \\
\text { / Bastante / Mucho / } \\
\text { Muchísimo }\end{array}$ \\
\hline 5 & $\begin{array}{l}\text { ¿Quieres añadir algo que nos sirva para mejorar el vídeo o el } \\
\text { juego? }\end{array}$ & Respuesta abierta. \\
\hline
\end{tabular}

Fuente: elaboración propia

Ambos instrumentos han sido elaborados de manera conjunta entre los investigadores actuando como un primer juicio de expertos. La intención es que el propio prepilotaje del cuestionario sirviera como primera prueba de validez y que sentara las bases del ulterior cuestionario a desarrollar en la fase de pilotaje.

\subsection{Procedimiento de la investigación}

Tras la fase de aplicación, se definieron las variables y se conformó la matriz de datos a través del programa IBM SPSS Statistics for Windows (ver. 21). Se procedió a un análisis descriptivo univariable, consistente en el cálculo de estadísticos descriptivos, frecuencias y porcentajes para cada una de las variables. Por su parte, para la creación de la red semántica relativa a la percepción sobre lo aprendido se empleó el programa de análisis cualitativo Atlas ti. for Windows (ver. 6.2). La tabla de códigos empleada para el análisis cualitativo queda reflejada en la tabla 3. 
Tabla 3

Tabla de Códigos

\begin{tabular}{|c|c|c|}
\hline 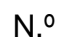 & Código & Comentarios \\
\hline 1 & Aprendido-Juego & $\begin{array}{l}\text { Menciones que hacen referencia a elementos } \\
\text { aprendidos al jugar. }\end{array}$ \\
\hline 2 & Aprendido-Vídeo & $\begin{array}{l}\text { Menciones que hacen referencia a elementos } \\
\text { aprendidos al ver el vídeo introductorio. }\end{array}$ \\
\hline 3 & Escudo de la Orden & $\begin{array}{l}\text { Menciones que recuerdan el escudo de la Orden } \\
\text { de Santiago. }\end{array}$ \\
\hline 4 & Época y reyes & $\begin{array}{l}\text { Menciones referidas a la época en la que se } \\
\text { ambienta el juego y a los reyes que gobernaban } \\
\text { en esa época. }\end{array}$ \\
\hline 5 & Función de la Encomienda & $\begin{array}{l}\text { Menciones referidas a la función de la Encomienda } \\
\text { de Ricote. }\end{array}$ \\
\hline 6 & Moriscos & Menciones referidas a los moriscos. \\
\hline 7 & Nombre de la Orden & $\begin{array}{l}\text { Menciones referidas al nombre de la Orden que } \\
\text { construyó la Encomienda de Ricote. }\end{array}$ \\
\hline 8 & Observaciones & $\begin{array}{l}\text { Menciones que hacen algún tipo de sugerencia o } \\
\text { propuesta de mejora del juego. }\end{array}$ \\
\hline 9 & Ricote & Menciones referidas a la población de Ricote. \\
\hline 10 & Sobre el juego & $\begin{array}{l}\text { Opiniones sobre el juego, sin realizar propuesta de } \\
\text { cambio o mejora. }\end{array}$ \\
\hline
\end{tabular}

Fuente: elaboración propia

\section{RESULTADOS}

\subsection{Objetivo 1. ¿Qué contenidos recuerdan los estudiantes?}

Para responder a este objetivo, se procedió a realizar un análisis cuantitativo de las respuestas dadas al cuestionario $\mathrm{C} 1$ (en sus fases de pre-test y post-test) así como un análisis de corte cualitativo de los ítems 1 y 2 del instrumento C2.

La aplicación del cuestionario C1 como pre-test y post-test estaba dirigido a detectar qué aprendizajes se asociaban al juego. Los datos confirman que tras el post-test los estudiantes aumentaron sus conocimientos. Sin embargo, interesaba constatar en qué ítems en concreto este avance había sido realmente significativo (Tabla 4).

Dichos resultados coinciden a grandes rasgos con la propia apreciación del alumnado, tal y como reflejan las respuestas obtenidas del análisis cualitativo del cuestionario C2 (Figura 1).

Los estudiantes fueron capaces de distinguir entre los elementos que habían aprendido en el vídeo y el juego. Atendiendo a las respuestas del alumnado participante, los contenidos históricos propiamente dichos se concentran en mayor medida en lo relativo al vídeo, mientras 
que cuando analizan los contenidos asociados al juego se reduce su sensación de haber aprendido contenidos más disciplinares, y pasan a destacar los relacionados con un aprendizaje operacional, es decir, aquellos inherentes a la propia dinámica del juego.

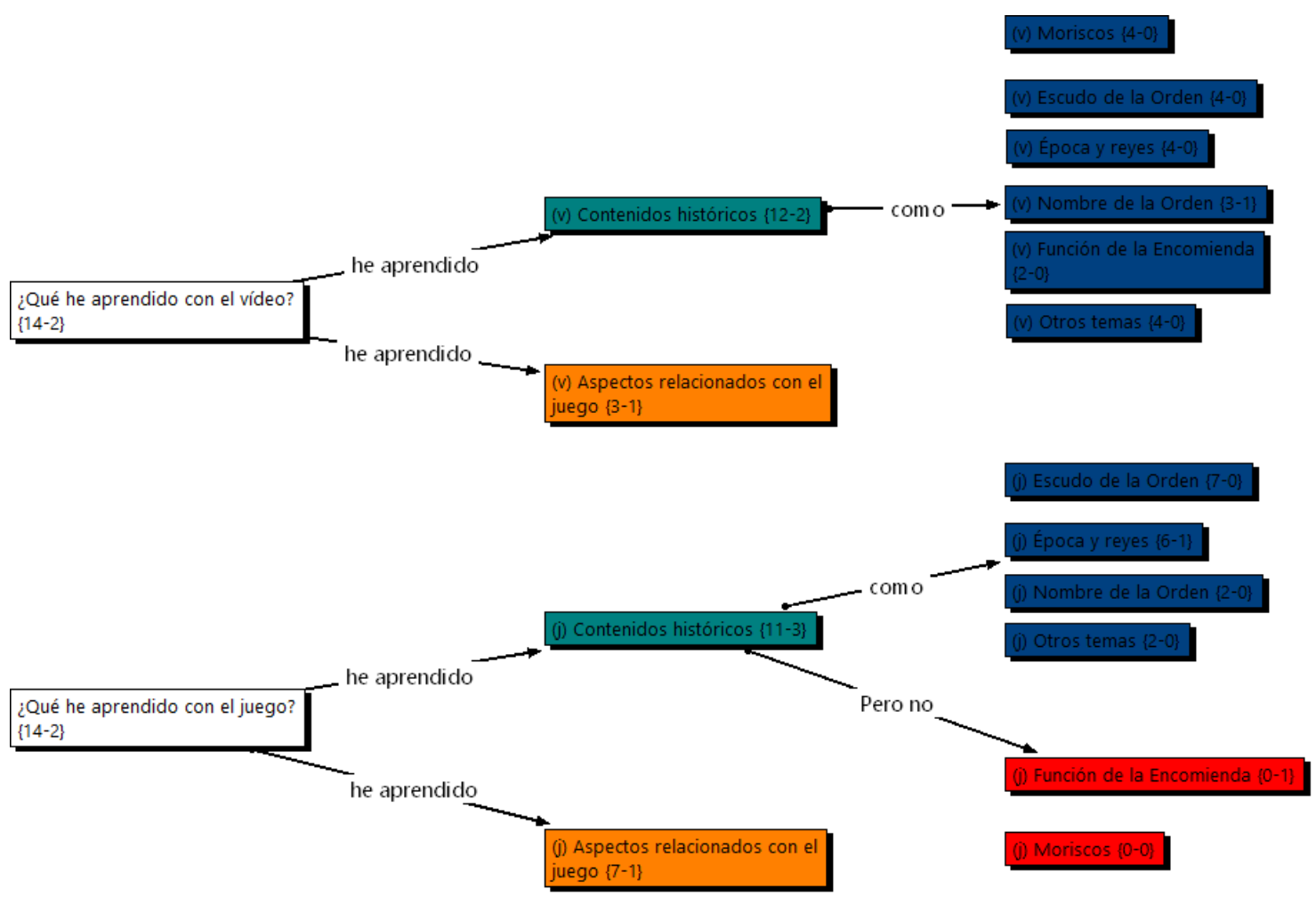

Figura 1. Red Semántica “¿Qué afirman los estudiantes que han aprendido?

\subsection{Objetivo 2. ¿Qué tipo de pruebas son más propicias a que se recuerden los contenidos?}

Las respuestas anteriores llevan a relacionar aquello que recuerda el alumnado con el tipo de prueba al que cada uno de estos conceptos se encuentra asociada, intentando determinar así la relación entre aprendizaje y estrategia utilizada.

Como era de suponer, si se comparan los resultados entre el pre-test y el post-test los estudiantes mejoraron todas sus respuestas. Se trataba de un tema en el que, como se puede apreciar, muchos tenían poca información.

A tenor de los resultados mostrados, la presencia de contenidos narrados en el vídeo se muestra fundamental a la hora de transmitir información, aunque muchos de ellos no vayan acompañados de ningún tipo de apoyo visual. Sin embargo, cuando los contenidos se reiteran a través de diferentes recursos parece reforzarse el aprendizaje, como así se observa en el ítem 5 (Tabla 4). 
Tabla 4

Nivel de acierto del cuestionario 1 y estrategia utilizada para presentar cada contenido

\begin{tabular}{|c|c|c|c|c|c|}
\hline \multirow{2}{*}{ Ítem } & \multicolumn{2}{|c|}{ Estrategia utilizada } & \multicolumn{2}{|c|}{ Frecuencias } & \multirow[b]{2}{*}{ Diferencia } \\
\hline & vídeo & videojuego & Pre-test & Post-test & \\
\hline 1 (Ubicación geográfica) & - & - & $68.18 \%$ & $80 \%$ & $+11.82 \%$ \\
\hline 2 (Función edificio) & Narración & - & $0 \%$ & $21,43 \%$ & $+21.43 \%$ \\
\hline 3 (Cronología) & Narración & $\begin{array}{l}\text { Búsqueda - } \\
\text { Imagen }\end{array}$ & $27.27 \%$ & $57.14 \%$ & $+29.87 \%$ \\
\hline 4 (Nombre Orden) & Narración & Narración & $0 \%$ & $28.57 \%$ & $+28.57 \%$ \\
\hline 5 (Escudo Orden) & $\begin{array}{l}\text { Narración } \\
\text { Imagen }\end{array}$ & $\begin{array}{c}\text { Elección } \\
\text { múltiple } \\
\text { (imágenes) } \\
\text { Puzzle } \\
\text { (imagen) }\end{array}$ & $22.73 \%$ & $100 \%$ & $+77.27 \%$ \\
\hline 6 (Moriscos) & Narración & - & $27.27 \%$ & $78.57 \%$ & $+51.3 \%$ \\
\hline 7a (Identifica Carlos I) & - & - & $9.09 \%$ & $14.29 \%$ & $+5.2 \%$ \\
\hline 7b (Identifica RRCC) & - & $\begin{array}{l}\text { Elección } \\
\text { múltiple } \\
\text { (imágenes) }\end{array}$ & $13.64 \%$ & $35.71 \%$ & $22.07 \%$ \\
\hline
\end{tabular}

Fuente: elaboración propia

\subsection{Objetivo 3. ¿Cuál es la valoración de los estudiantes?}

Además del propio análisis de las respuestas obtenidas, resultaba interesante conocer las valoraciones que los propios estudiantes otorgan a la acción diseñada (juego y vídeo) desde un punto de vista lúdico y educativo. Respecto a la satisfacción con lo aprendido (C2, Ítem 3) y con el juego (C2, Ítem 4) los resultados son bastante elevados. Si se comparan las medias de ambos ítems se observa que la satisfacción con el juego (=5,7 sobre 6$)$ es mayor que la satisfacción con lo aprendido (=4 sobre 6) (Tabla 5) a pesar de que en el análisis cualitativo las menciones a elementos aprendidos en el vídeo son mayores.

Tabla 5

Nivel de satisfacción de los alumnos con lo aprendido gracias al juego y con el propio juego

\begin{tabular}{ccccc}
\hline & Muy poco & Poco & Bastante & Muchísimo \\
\hline Satisfacción con lo aprendido & $7.1 \%$ & $14.3 \%$ & $64.3 \%$ & $14.3 \%$ \\
Satisfacción con el juego & - & - & $28.6 \%$ & $71.4 \%$ \\
\hline
\end{tabular}




\section{DISCUSIÓN Y CONCLUSIONES}

Aunque como ya se dijo el uso de videojuegos conlleve un aprendizaje per se (Becker, 2011) y se esté favoreciendo una alfabetización digital (Gee, 2007), el juego por el hecho de serlo no tiene porqué favorecer el aprendizaje de contenidos más conceptuales. En este caso, en el que cabe recordar que no se trataba de un videojuego educativo al uso, los datos preliminares, a falta de la fase de pilotaje, permiten comprobar que el alumnado sí que es capaz de recordar muchos de los contenidos introducidos a lo largo de la acción educativa propuesta. De hecho, en todas las pruebas se produce un aumento de las respuestas correctas. Se trata eminentemente de contenidos de tipo conceptual muy concretos, como quiénes eran los moriscos o cuál era la función del edificio, a excepción hecha del aprendizaje operacional directamente ligado a la propia habilidad manipulativa y que ha sido destacado por el conjunto del alumnado.

En relación a las estrategias que han permitido esos aprendizajes, es curioso observar que algunas de las pruebas en las que el aprendizaje ha crecido más están relacionadas con los contenidos vistos en el vídeo. Tanto la "función del edificio" como el significado de "moriscos" recientemente comentado únicamente aparecían en la introducción audiovisual y de forma narrada (Tabla 4). Es decir que, a pesar de lo que se podría pensar en un inicio, la información del vídeo introductorio es capaz de llegar al alumnado, puesto que entienden que gracias a esos datos tendrán más posibilidades de jugar correctamente. Sin embargo, parece que el recuerdo mayor se consigue en elementos que se trabajan tanto en el juego como en el vídeo y que, además, son elementos que se presentan de manera visual, como es el caso de la cronología del edificio (señalada en el audio y sobreimpresa en la columna interior del patio de la casa) o el escudo de la orden (descrito en el audio y representada en una de las pruebas del videojuego). Concretamente este último es recordado por la totalidad de los jugadores. Parece que lo visual facilita el recuerdo y permite reforzar aquello que ha sido previamente introducido de manera narrativa y/o descriptiva.

Junto a todo esto, la receptividad de los estudiantes hacia el juego fue muy alta, lo que resulta completamente lógico pues se les está ofreciendo algo que encaja perfectamente con su propia cultura lúdica, en el sentido del término defendido por Brougère (2005, 2013). La elevada receptividad hacia el juego debe ponerse en relación con el hecho de que durante unos minutos se sienten parte de un escenario, resuelven situaciones complejas o se enfrentan a momentos de incertidumbre, hechos que ya se han definido en otras ocasiones como elementos a tener en cuenta a la hora de diseñar productos digitales para las aulas (Garrido, 2013). En cuanto a la receptividad sobre lo aprendido (4 sobre 6), aunque elevada, es relativamente más baja si se compara con su opinión sobre el juego (5,7 sobre 6$)$. A modo de ejemplo de la opinión sobre el juego, una participante decía que había aprendido "a utilizar un juego de realidad virtual, algo que me ha dejado alucinada" (Alumno 13). Sin embargo, aunque algunos apuntan que han aprendido contenidos como la cruz de la Orden de Santiago o la fecha de construcción, algún alumno se sincera y reconoce que "no aprendes más rápido porque tienes que estar concentrado" (Alumno 12). En este sentido, lo corto de la experiencia y la celeridad con la que tienen que resolver las pruebas va en contra del propio aprendizaje.

La investigación sigue abierta y, de hecho, en breve se podrán extraer los datos de la fase de pilotaje en el que se ha ampliado la muestra y se ha podido realizar un estudio estadístico. Por lo tanto, no se está en situación de generalizar ahora ningún tipo de resultado. Sin embargo, se trata de una investigación pionera en cuanto a la posibilidad de evaluar el aprendizaje de contenidos históricos a través de los videojuegos. En futuras fases de la 
investigación, con una mayor muestra, por ejemplo, se será capaz de poder aportar más datos que confirmen lo que aquí se plantea.

Sin embargo, con los primeros datos en la mano ya se observan ciertos elementos que permiten marcar una dirección clara para los nuevos productos a diseñar. A pesar de las dificultades que los videojuegos poseen a la hora de protagonizar estrategias de enseñanzaaprendizaje en los centros educativos, ya sea por su elevado coste, por su eminente carácter individual o por la dificultad de plantear actividades colaborativas (Feliu y Hernàndez, 2011), la aparición ocasional de este tipo de recursos en el aula aportan sin duda una alta carga motivacional al alumnado. A falta de un análisis más exhaustivo, los primeros datos sí que parecen comprobar que el videojuego actúa, en este caso, como un elemento claramente motivador más que intrínsecamente educador. Es sabido que el videojuego cuenta con ciertas cualidades propias que lo hacen muy llamativo en estas edades (Evaristo et al., 2016). Pero será necesario aprovechar este estímulo con la inclusión de pruebas en juegos venideros que realmente aporten elementos didácticamente más rentables, ya sean contenidos conceptuales o, por qué no, estrategias gamificadas que pueda favorecer que el estudiante aprenda a pensar históricamente (Seixas y Morton, 2013). De esta manera, se estarían potenciando determinados procedimientos relacionados con la historia 0 incluso introducir narrativas 0 elementos en el juego que estén aportando ciertos valores, como el respeto al patrimonio, la interculturalidad, etc.

\section{ENLACES}

Vídeo de introducción a El Misterio de la Encomienda de Ricote:

https://www.regmurcia.com/servlet/s.SI?METHOD=DETALLEMEDIATECA\&serv=Mediateca\&ml $\mathrm{d}=14331$

\section{RECONOCIMIENTOS}

Trabajo realizado en el marco del Proyecto "La evaluación de las competencias y el desarrollo de capacidades cognitivas sobre Historia en Educación Secundaria Obligatoria" (EDU2015-65621-C3-2-R), financiado por el Ministerio de Economía y Competitividad de España / FEDER, así como el contrato "Didáctica de las Ciencias Sociales aplicada a recursos digitales sobre el patrimonio de la Región de Murcia" entre la Universidad de Murcia y la Fundación Integra.

\section{REFERENCIAS BIBLIOGRÁFICAS}

Anderson, E. F., McLoughlin, L., Liarokapis, F., Peters, C., Petridis, P., y Freitas, S. de. (2010). Developing serious games for cultural heritage: a state-of-the-art review. Virtual Reality, 14(4), 255-275. doi: 10.1007/s10055-010-0177-3

Ayén, F. (2010). Los videojuegos en la didáctica de la historia. BITS. Revista de la Asociación Espiral, Educación y Tecnología, 18. Recuperado de www.index.html@p=65.html

Barbier, B. (2014). Video Games and Heritage: Amateur Preservation? Hybrid. Revue Des Arts et Médiations Humaines, 1. Recuperado de http://www.hybrid.univparis8.fr/lodel/index.php?id=287

Becker, K. (2011). The Magic Bullet: A Tool for Assessing and Evaluating Learning Potential in Games. International Journal of Game-Based Learning, 1(1), 19-31.

Brougère, G. (2005). Jouer/apprendre. Paris: Economica ; Anthropos.

Brougère, G. (2013). El niño y la cultura lúdica. Ludicamente, 2(4). 
Cuenca, J. M. (2001). Los juegos informáticos de simulación en la enseñanza y el aprendizaje de las ciencias sociales. Iber: Didáctica de las Ciencias Sociales, Geografía e Historia, 30, 69-82.

Cuenca, J. M., y Martín, M. J. (2010). La resolución de problemas en la enseñanza de las ciencias sociales a través de videojuegos. Iber: Didáctica de las Ciencias Sociales, Geografía e Historia, 63, 32-42.

Decreto n.․ 220/2015, de 2 de septiembre de 2015, por el que se establece el currículo de la Educación Secundaria Obligatoria en la Comunidad de Autónoma de la Región de Murcia. Boletín Oficial de la Región de Murcia (BORM), 203, pp. 30729-31593.

Del Moral, M. E., Villalustre, L., Yuste, R., y Esnaola, G. (2012). Evaluación y diseño de videojuegos: generando objetos de aprendizaje en comunidades de práctica. RED: Revista de Educación a Distancia, 33, 1-17.

Escribano-Miralles, A. (2016). La Encomienda de Ricote: un paseo por el siglo XVI. Iber: Didáctica de las ciencias sociales, geografía e historia, 85, 81-82.

Etxeberria, F. (2001). Videojuegos y educación. Education in the knowledge society (EKS), 2, 3.

Evaristo, I. S., Vega, M. V., Navarro, R., y Nakano, T. (2016). Uso de un videojuego educativo como herramienta para aprender historia del Perú. RIED. Revista Iberoamericana de Educación a Distancia, 19 (2), 35-52. doi: 10.5944/ried.19.2.15569

Feliu, M., y Hernàndez, F. X. (2011). 12 ideas clave: Enseñar y aprender historia. Barcelona: Graó.

Gálvez, M. del C. (2006). Aplicaciones de los videojuegos de contenido histórico en el aula. Revista ICONO14. Revista científica de Comunicación y Tecnologías emergentes, 4, 217230. doi: $\underline{10.7195 / \text { ri14.v4i1.405 }}$

Garrido Miranda, J. M. (2013). Videojuegos de estrategia: algunos principios para la enseñanza. Revista Electrónica de Investigación Educativa, 15(1), 62-74.

Gee, J. P. (2007). What video games have to teach us about learning and literacy (Rev. and updated ed). New York: Palgrave Macmillan.

Grup F9 (1998). Ciencias Sociales y juegos de ordenador: jugando con Carmen Sandiego. Cuadernos de Pedagogía, 289, 24-27.

Jiménez Alcázar, J. F. (2016). De la edad de los imperios a la guerra total: medievo y videojuegos. Murcia: Compobell.

Jiménez-Palacios, R., y Cuenca López, J. M. (2015). El uso didáctico de los videojuegos. Concepciones e ideas de futuros docentes de ciencias sociales. Clío: History and History Teaching, 41. Recuperado de http://clio.rediris.es/n41/articulos/JimenezCuenca2015.pdf

Jiménez-Palacios, R., y Cuenca López, J. M. (2016). Construyendo el conocimiento a través de videojuegos. En L. Arias Ferrer, A. I. Ponce Gea, y D. Verdú González (Eds.), Estrategias y recursos para la integración del patrimonio y los museos en la educación formal (pp. 237247). Murcia: Editum.

McMillan, J. H., y Schumacher, S. (2005). Investigación educativa: una introducción conceptual. Madrid: Pearson.

Moreno Castañeda, M. (2010). Aprender Historia en ambientes virtuales. Tejuelo, 9(1), 58-82.

Pérez, P., y Molina, J. M. (Directores). (2011). Carthago Nova [Película]. España: Fundación Integra.

Rivero, M. P. (2009). El aprendizaje del mundo romano: fuentes de conocimiento no formal del alumnado de secundaria. Didáctica de Las Ciencias Experimentales Y Sociales, 23, 61-69. doi: $10.7203 /$ dces..2406

Seixas, P., y Morton, T. (2013). The big six: historical thinking concepts. Toronto: Nelson Education.

Téllez, D., y Iturriaga, D. (2014). Videojuegos y aprendizaje de la Historia: la saga Assasin's Creed. Contextos educativos, 17, 145-155. 


\title{
INFORMACIÓN SOBRE LOS AUTORES
}

\author{
Alejandro Egea Vivancos \\ Universidad de Murcia
}

Licenciado en Historia Antigua y Arqueología por la Universidad de Murcia (1998), licenciado en Antropología Social y Cultural por la UNED (2007) y doctor por la Universidad de Murcia (2003). Ha ampliado estudios en centros de investigación sirios (Damasco, Alepo), ingleses (Londres), alemanes (Heidelberg, Munich, Bonn), italianos (Roma) y norteamericanos (Lexington, KY). Tras diez años ejerciendo como investigador en Historia Antigua en la Universidad de Murcia, seis años como Profesor de Educación Secundaria y dos como Profesor Asociado en el Área de Didáctica de las Ciencias Sociales de la Facultad de Educación de la Universidad de Murcia, desde 2014 trabaja en exclusividad en la citada facultad. Compagina la investigación con su docencia en el Grado de Educación Primaria, el Máster de Formación del Profesorado y el Máster de Historia y Patrimonio. Actualmente centra su línea de investigación en la educación patrimonial, con especial atención a la incorporación del patrimonio cultural y en concreto la arqueología en las aulas. Miembro del Grupo de Investigación DICSO y Subdirector del CEPOAT. Web: https://webs.um.es/alexegea

\section{Laura Arias Ferrer \\ Universidad de Murcia}

Licenciada en Historia por la Universidad de Murcia, y doctora por la Universidad de Alicante, actualmente es Profesora Contratada Doctora en el Departamento de Didáctica de las Ciencias Matemáticas y Sociales de la Facultad de Educación de la Universidad de Murcia. Previamente pudo desarrollar su labor como docente e investigadora en la Universidad de Murcia, merced a una beca de investigación predoctoral y a un posterior contrato posdoctoral. Su vinculación con el campo de la didáctica se vio reforzado con su estrecha colaboración con los departamentos de didáctica del Museo de Arte Ibérico del Cigarralejo (Mula, Murcia) y del Museo Arqueológico de Murcia. Actualmente centra su trabajo en innovación en la práctica docente, en la formación del profesorado de educación infantil y primaria, así como en el análisis de recursos para la enseñanza de la historia en distintos niveles educativos, con especial atención al relacionado con el patrimonio cultural. Miembro del Grupo de Investigación DICSO y Secretaria del CEPOAT. Web: https://webs.um.es/larias

\section{Alfonso J. García López Fundación Integra}

Ingeniero Superior en Informática por la Universidad de Murcia. Trabajó como programador especialista en Internet para la Universidad de Murcia y como asesor en gestión de contenidos Web para la Comunidad Autónoma de la Región de Murcia. En 2001 fue coautor del concepto de Portal Regional de Murcia, hoy denominado Región de Murcia Digital, www.regmurcia.com. Desde 2003 trabaja como Jefe de Proyectos de la Fundación Integra como responsable de contenidos multimedia de Región de Murcia Digital. Desde 2006 se ha encargado de la dirección de más de 30 proyectos de producción audiovisual de carácter documental sobre el Patrimonio de la Región de Murcia, destacando especialmente por su complejidad y singularidad el proyecto dedicado a Carthago Nova, finalista en la categoría de "Mejor Película de Animación" en los Premios Goya 2012.

\section{(cc) EY-No}

Los textos publicados en esta revista están sujetos a una licencia de Reconocimiento 4.0 España de Creative Commons. Puede copiarlos, distribuirlos, comunicarlos públicamente y hacer obras derivadas siempre que reconozca los créditos de las obras (autoría, nombre de la revista, institución editora) de la manera especificada por los autores o por la revista. La licencia completa se puede consultar en:Licencia Creative Commons Atribución-NoComercial-Compartir por igual 4.0 Internacional. 